\title{
Chiral recognition of polysaccharide based CSP for separation of enantiomers and regio isomers of Prasugrel and its related impurities
}

\author{
Vuyyuru Nagireddy ${ }^{\mathrm{a}, \mathrm{b}}$, G. Vamsi krishna ${ }^{\mathrm{a}}$, V. Malati ${ }^{\mathrm{a}}$, B.Ramadevi ${ }^{\mathrm{b}}$, Y. Ravindra kumar ${ }^{\mathrm{a}}$ \\ ${ }^{a}$ Active Pharmaceutical Ingredients, Dr. Reddy's Laboratories Ltd. IPDO, Bachupally, Hyderabad-500090, T.S, India. \\ bJNTUH College of Engineering, J.N.T.University, Kukatpally, Hyderabad-500072, T.S, India.
}

\begin{tabular}{l} 
ARTICLE INFO \\
\hline Article history: \\
Received on: $24 / 04 / 2017$ \\
Accepted on: 09/06/2017 \\
Available online: $30 / 07 / 2017$ \\
\hline Key words: \\
Chiral stationary phase \\
(CSP), LUX Amylose-2, \\
Prasugrel, Regio isomers, \\
Organic modifiers, photo \\
diode array detector (PDA).
\end{tabular}

\begin{abstract}
The current study focuses on the efficiency of chiral chromatographic conditions for separation of critical enantiomers and regio-isomeric pairs of Prasugrel, an antiplatelet drug and its related impurities. Totally, 10 chiral compounds were screened and well resolved using Phenominex-LUX-2-Amylose based chiral HPLC column (polysaccharide CSP). Chiral separations were achieved using Isopropanol, ethanol as organic modifiers and trimethylamine (TEA), trifluoro acetic acid (TFA) as polar modifier. Impact assessment for selection of superior chromatographic conditions to achieve best resolution was evaluated by varying column temperatures and concentrations of organic and polar modifiers. Significant enantiomeric separations were achieved with low alcohol and high TFA concentrations as modifiers in mobile phase. With optimized conditions, not only Prasugrel's enantiomers, two process related impurities (3-floro and 4-floro prasugrel) and major degradant i.e., Des acetyl impurity which exhibits keto-enol tautomerism in solution state were also separated into respective individual enantiomeric forms. Hence, the current work can be implemented for evaluation of chiral impurities of Prasugrel during synthesis as well as in Active Pharmaceutical Ingredient.
\end{abstract}

\section{INTRODUCTION}

Recently, optically active compounds gained attention towards various fields including pharmaceutics, agrochemicals, ferroelectric, liquid crystals, etc., and therefore their preparation, identification and analysis became increasingly important (Lein et al., 2007, Ulrich et al., 2012). Recent Advances in optical resolution and chiral separations by high-performance liquid chromatography (HPLC) has progressed rapidly, and has become a powerful methodology whose applications were not only limited for determining optical purity but also for purification of optical isomers (Stevenson et al., 1991). It is well known that biological systems are specific in chiral recognitions and impose significant impact on drug's pharmacological and toxicological responses (Waldeck et al., 1993, Lein Ai et al., 2006).

\footnotetext{
* Corresponding Author

E-mail:nagirv@drreddys.com,nagirv@gmail.com Tel.:040 44346307, +91994920527
}

Prasugrel is a racemic mixture and chemically described as (RS)-5-[2-cyclopropyl-1-(2-fluorophenyl)-2-oxoethyl]-4,5,6,7tetrahydrothieno [3,2-c]pyridin-2-yl acetate. It is a new oral thienopyridine anti-platelet drug from the same class as clopidogrel and has a more rapid and potent effect on platelet inhibition than does clopidogrel (Bhatt et al., 2003, Z.-M. Wang et al., 2010). It has an empirical formula of $\mathrm{C}_{20} \mathrm{H}_{20} \mathrm{FNO}_{3} \mathrm{~S}$, a molecular weight of $373.44 \mathrm{~g} /$ mole. Both the prasugrel's isomers have same biological activity and active metabolites inhibits platelet activation and aggregation by irreversibly blocking P2Y12 adenosine diphosphate receptors. Prasugrel is also approved for treating acute coronary syndrome (moderate to high risk unstable angina, ST-segmentelevation myocardial infarction (STEMI) or non-ST-segmentelevation myocardial infarction (NSTEMI) in combination with aspirin in people who are to undergo percutaneous coronary intervention (PCI). As per thorough literature survey, few research articles were published till date which discusses about quantification of prasugrel and its enantiomeric metabolites in human plasma. 
One method discusses about Stereo selective Metabolism of Prasugrel in Humans Using a Novel Chiral Liquid Chromatography-Tandem Mass Spectrometry Method (Enaksha et al., 2007). Another work reveals about Electrospray ionization LC-MS/MS validated method for the determination of the active metabolite (R-138727) of prasugrel in human plasma and its application to a bioequivalence study (Ojikumar et al., 2011). Few more research articles discusses about impurity profiling and comparison of forced degradation behavior of prasugrel by HPLC and UPLC (Singh et al., 2015, Malati et al., 2012, Kapendra et al., 2011). But, till date no work represents enantiomeric separation of prasugrel in active pharmaceutical ingredient's perspective, which basically add core value to manufacturing sector. No Official monograph is available at present, for drug substance and drug product which supports chiral separation of prasugrel.

Hence, the aim of our study is to develop a chromatographic method to separate racemic mixture of prasugrel and its positional isomers as well as its process related impurities and major degradant into individual enantiomers. The effect of the steric structure of selected CSP and concentration of the mobile phase modifier on the retention (k') and stereo selectivity $(\alpha)$ of a series of prasugrel related enantiomers along with impact of column temperature on retention and resolution were studied and represented in this work.

\section{MATERIALS AND METHOD}

\section{Chemicals and Reagents}

Samples of Prasugrel, isomers and desacetyl impurity in its racemic forms with $>95 \%$ purity were supplied by Process Research Department of Integrated product development operations of Dr. Reddy's Laboratories Limited, Hyderabad, India. HPLC-grade n-hexane, 2-propanol, ethanol were purchased from
S.D. Fine chem. (Mumbai, India). Triethylamine was purchased from J. T. Baker (Phillipsburg, NJ, U.S.A.) and trifluoroacetic acid (TFA) was of analytical grade from Sigma-Aldrich (Madrid,Spain).

\section{Instrumentation and Chromatographic conditions}

Method development and verification studies were carried on Waters alliance 2489 HPLC system equipped with a photo diode array detector (PDA). The output signal was monitored at UV wavelength of $215 \mathrm{~nm}$ and data processed using Empower-2 software. The chromatographic separation was achieved on a Lux amylose-2, $250 \mathrm{~mm}$ x $4.6 \mathrm{~mm}, 5 \mu \mathrm{m}$ column supplied by Phenomenex, USA, using a mobile phase containing n-hexane - ethanol - TEA - TFA (90: $10: 0.1: 0.2, \mathrm{v} / \mathrm{v} / \mathrm{v} / \mathrm{v})$. The flow rate of the mobile phase was kept at $1.0 \mathrm{~mL} / \mathrm{min}$ in iso-cratic mode. The column oven temperature was maintained at $35^{\circ} \mathrm{C}$ and the injection volume was set at $10 \mu \mathrm{L}$.

\section{Test Sample preparation}

Absolute Ethanol was used as diluent during the test samples preparation. Prior to sample preparation, glassware used for study were thoroughly washed and rinsed with diluent to restrict contamination. Weighed and transferred appropriate quantities of racemic mixtures of Prasugrel (PRA-E1 and PRAE2), 3-fluoro impurity (PRA-3F-E1 and PRA-3F-E2) and 4-fluoro impurity (PRA-4F-E1 and PRA-4F-E2) to a $10 \mathrm{~mL}$ amber volumetric flask. Vortexed to dissolve completely and further dilutions were made with ethanol to meet final concentration of $0.8 \mathrm{mg} / \mathrm{mL}$. This initial concentration was chosen to evaluate the elution pattern of selected compounds. Refer to Figure-1 for structural details of compounds.

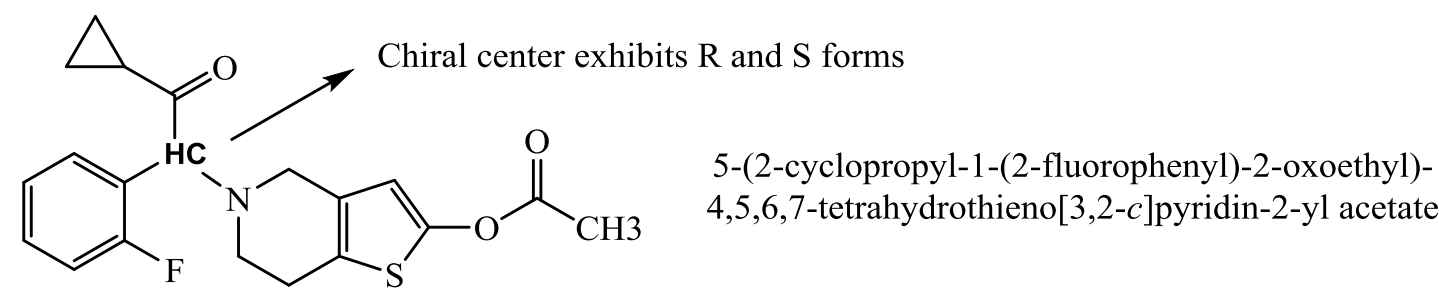

Compound -1 and 2 (PRA-E1 and PRA-E2)

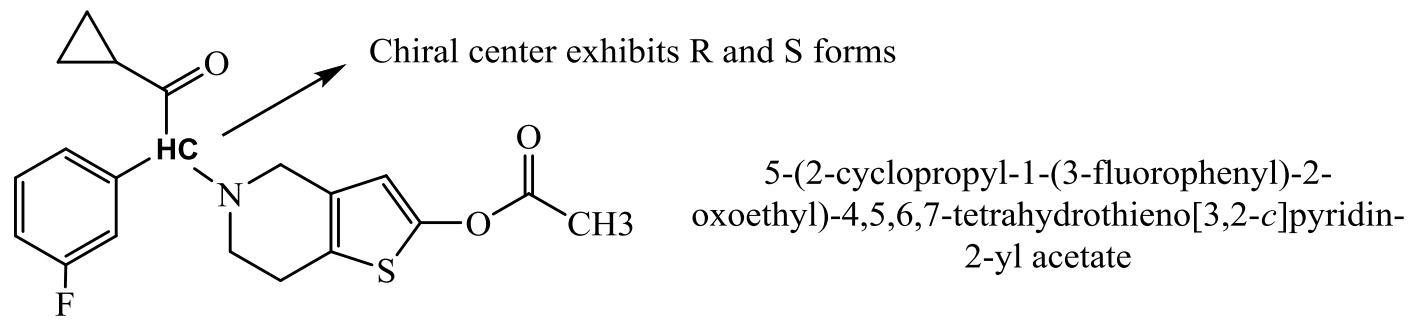

Compound -3 and 4 (PRA-3F-E1 and PRA-3F-E2) 


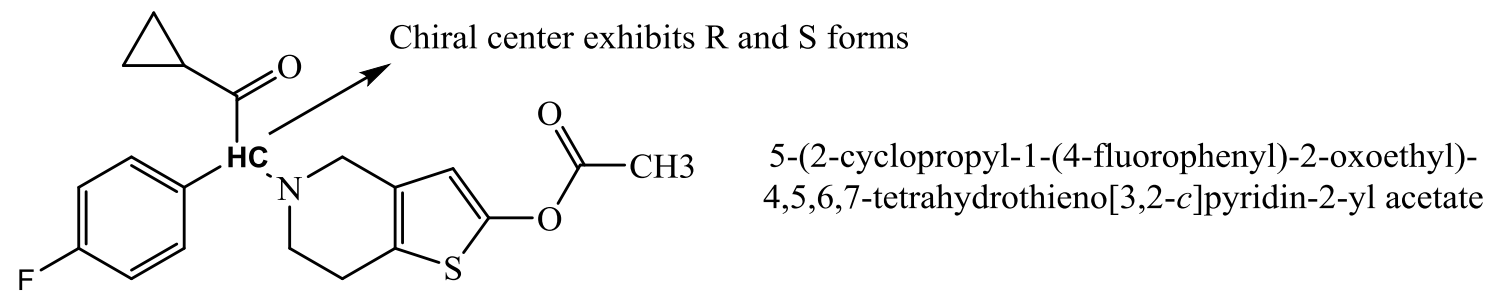

\section{Compound -5 and 6 (PRA-4F-E1 and PRA-4F-E2)}

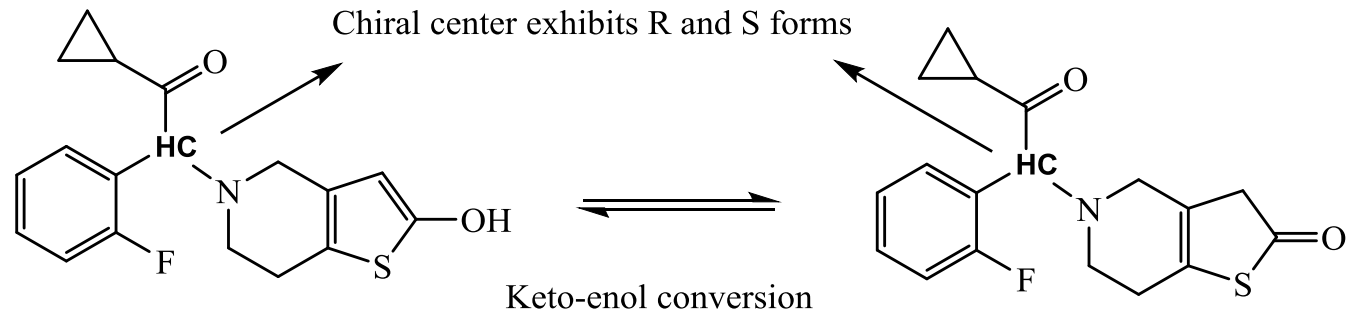
Compound -7, 8, 9 and 10 (PRA-DA-E1, 2, 3, 4) 1-cyclopropyl-2-(2-fluorophenyl)-2-(2- hydroxy-6,7-dihydrothieno[3,2- c]pyridin-5(4H)-yl)ethan-1-one

Fig. 1: Prasugrel and its related enantiomers and regio isomers.

\section{RESULTS AND DISCUSSIONS}

\section{Optimization of chromatographic conditions}

Several authors demonstrated that the acidic and basic mobile phase additives can have a significant impact in addition to the classical factors such as temperature, $\mathrm{pH}$ and type of organic modifier, which can influence the enantio separation in LC (Matthijs et al., 2006, Perrin et al., 2002, Ye Y et al., 2001, Levin et al., 1993, Khatuna et al., 2015, Gübitz et al., 2006). These studies mostly carried by normal-phase chromatography, as these additives were supposed to minimize the non-specific interactions between the analytes and the free silanol groups of the CSP. Hence study design was done by selecting normal-phase mode containing n-Hexane as major solvent, ethanol, isopropanol as organic modifiers and triethyl amine (TEA) and trifluoroacetic acid (TFA) as polar modifiers as main constituents of the mobile phase. Initial trials were performed to select suitable chiral recognition using available polysaccharide-based CSPs. Chiralcel OD-H (Cellulose tris (3,5-dimethylphenylcarbamate) and other amylose based columns like Chiralpak-IA (Immobilised Amylose tris-(3,5dimethylphenylcarbamate) and Chiralcel AD-H (Amylose tris(3,5-dimethylphenylcarbamate) were screened, which resulted in improper separation between certain pair of the enantiomers.
Lux amylose-2 column which possess amylose tris(5-chloro-2methylphenyl carbamte) as function group has shown good selectivity over the other amylose based column, where promising results were noticed in respect to resolution and peak shape.

Prior to testing, HPLC system was well equilibrated with chromatographic conditions at least for 3 hours, and baseline was monitored. Desacetyl impurity being major degradant exists in racemic mix like prasugrel, but exhibits keto-enol tautomerism in solution state (PRA-DA-1,2,3,4).

\section{Effect of the column temperature}

The influence of the temperature on the enantioresolution (Rs) of the studied chiral drugs was investigated. Based on observations, the change in enantioresolution with column temperature seems to be depend on chiral center of compound. For those which exhibited an increased enantioresolution with temperature, a significant efficiency enhancement was observed at $35^{\circ} \mathrm{C}$. However, low enantioresolution is observed at $25^{\circ} \mathrm{C}$ and $45^{\circ} \mathrm{C}$. Hence, was decided to select $35^{\circ} \mathrm{C}$ and the separation found unaffected at $\pm 3^{\circ} \mathrm{C}$ of finalized temperature. The results are illustrated in Table-1, figure-5. 
Table-1: Effect of temperature on chiral selectivity.

\begin{tabular}{|c|c|c|c|c|c|c|c|c|c|}
\hline & \multicolumn{3}{|c|}{$25^{\circ} \mathrm{C}$} & \multicolumn{3}{|c|}{$35^{\circ} \mathrm{C}$} & \multicolumn{3}{|c|}{$40^{\circ} \mathrm{C}$} \\
\hline & Rs & $\mathrm{k}^{\prime}$ & $\alpha$ & Rs & $\mathrm{k}^{\prime}$ & $\alpha$ & Rs & $\mathrm{k}^{\prime}$ & $\alpha$ \\
\hline Compound-3 & ----- & 15.9 & ----- & ----- & 12.52 & ----- & ----- & 7.96 & ----- \\
\hline Compound-4 & 2.31 & 17.98 & 1.13 & 2.38 & 13.98 & 1.12 & 2.27 & 8.93 & 1.12 \\
\hline Compound-5 & 1.14 & 19.14 & 1.06 & 1.32 & 14.88 & 1.06 & 1.23 & 9.51 & 1.06 \\
\hline Compound-6 & 2.69 & 21.75 & 1.14 & 1.87 & 16.25 & 1.09 & 1.82 & 10.44 & 1.10 \\
\hline Compound-1 & 1.01 & 22.76 & 1.05 & 1.49 & 17.48 & 1.08 & 1.38 & 11.23 & 1.08 \\
\hline Compound-2 & 4.83 & 30.99 & 1.36 & 5.38 & 23.07 & 1.32 & 4.97 & 14.87 & 1.32 \\
\hline
\end{tabular}

Rs = USP Resolution, $\mathrm{k}^{\prime}=\mathrm{K}$ prime (capacity factor), $\alpha=$ selectivity.

Influence of temperature on resolution

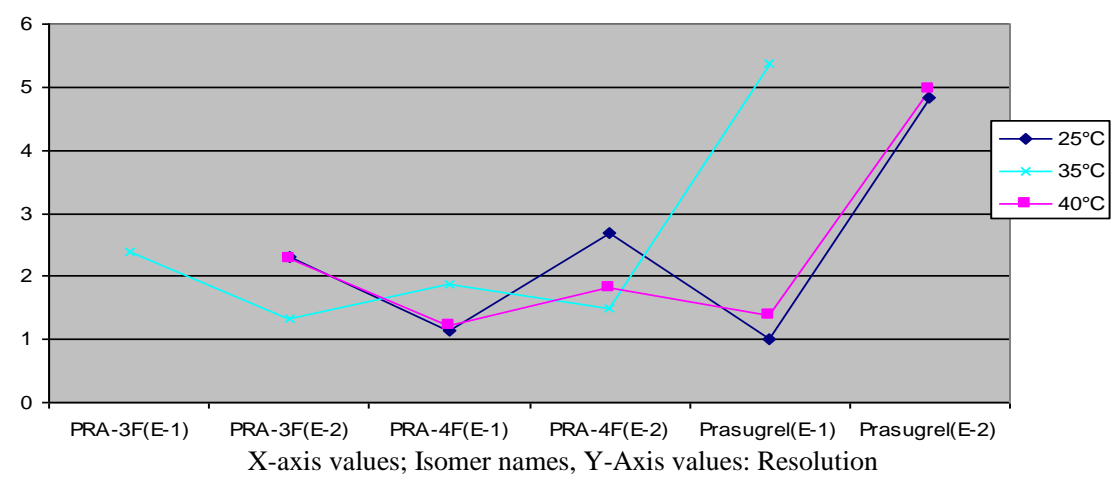

Fig. 5: Graphical representation of effect of column temperature on chiral selectivity.

Table-2: Influence of TFA Concentration on resolution.

\begin{tabular}{|c|c|c|c|c|c|c|c|c|c|}
\hline & \multicolumn{3}{|c|}{$1.0 \mathrm{~mL}$} & \multicolumn{3}{|c|}{$1.5 \mathrm{~mL}$} & \multicolumn{3}{|c|}{$2.0 \mathrm{~mL}$} \\
\hline & Rs & $\mathrm{k}^{\prime}$ & $\alpha$ & Rs & $\mathrm{k}^{\prime}$ & $\alpha$ & Rs & k' & $\alpha$ \\
\hline Compound-3 & ----- & 11.44 & $\begin{array}{l}---- \\
--1\end{array}$ & ----- & 15.9 & $\begin{array}{l}---- \\
--1\end{array}$ & ----- & 12.52 & ---- \\
\hline Compound-4 & 1.15 & 12.54 & 1.10 & 2.23 & 17.98 & 1.13 & 2.38 & 13.98 & 1.13 \\
\hline Compound-5 & 1.37 & 13.1 & 1.05 & 1.11 & 19.14 & 1.06 & 1.32 & 14.88 & 1.07 \\
\hline Compound-6 & 2.44 & 14.42 & 1.10 & 2.21 & 21.75 & 1.14 & 1.87 & 16.25 & 1.15 \\
\hline Compound-1 & 1.28 & 15.60 & 1.08 & 1.67 & 22.76 & 1.05 & 1.49 & 17.48 & 1.08 \\
\hline Compound-2 & 3.18 & 19.16 & 1.23 & 4.18 & 30.99 & 1.36 & 5.38 & 23.07 & 1.37 \\
\hline
\end{tabular}

Rs = USP Resolution, $\mathrm{k}^{\prime}=\mathrm{K}$ prime (capacity factor), $\alpha=$ selectivity.

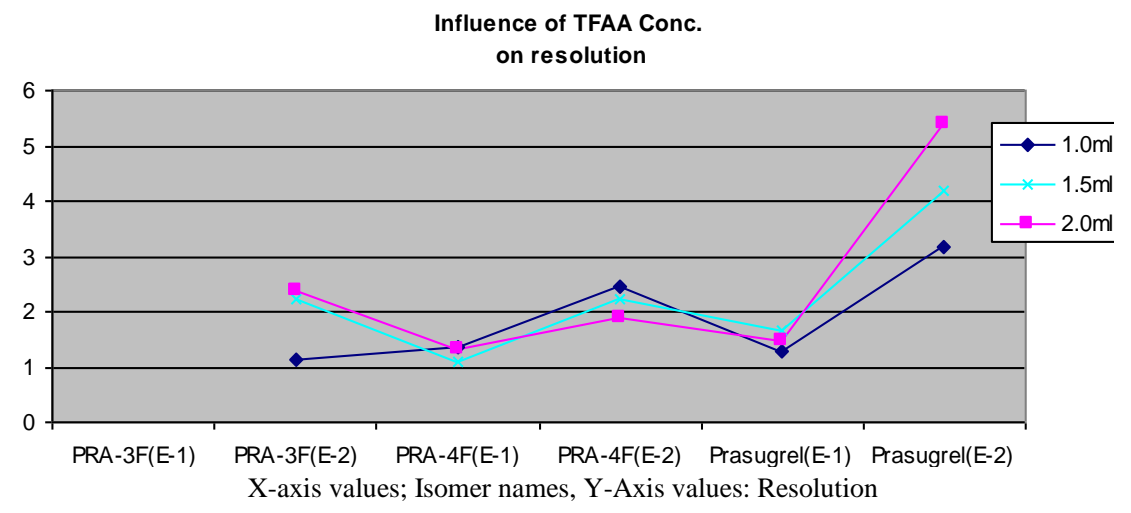

Fig. 6: Graphical representation of Influence of TFA Concentration on resolution.

\section{Effect of proportion of the acidic additive (TFA)}

Acidic additives has significant impact on enantioresolution and selectivity. Addition of Triflouroacetic acid (TFA) to the mobile phase yields separation between the isomers, this may be due to the ion-dipole interactions. It can be assumed that the introduction of chlorine on the phenyl moiety of Lux-2 CSP is supposed to reduce non-specific interactions with CSP in presence of acidic additive in the mobile phase.
During development, enantio resolution and selectivity were found to increase with increase in acidic additive concentration. As \% TFA in mobile phase found directly proportional to increased interactions, longer retention times for isomer peaks were observed. Therefore, This might be explained by a The results are illustrated in Table- 2 and figure- 6 which evaluates the influence of the proportion of TFA on the enantio resolution and selectivity within a range of $1.0 \mathrm{ml} / \mathrm{L}$ to $2.0 \mathrm{ml} / \mathrm{L}$. 
Table 3: Effect of Ethanol (Polar organic modifier) concentration on retention times.

\begin{tabular}{cccccccc}
\hline S.No & Conc. Of Ethanol & Compound-1 & Compound-2 & Compound-3 & Compound-4 & Compound-5 & Compound-6 \\
\hline $\mathbf{1}$ & $5.0 \%$ & 28.1 & 31.78 & 18.46 & 21.17 & 22.99 & 23.97 \\
$\mathbf{2}$ & $7.5 \%$ & 20.04 & 24.79 & 13.97 & 16.27 & 16.27 \\
$\mathbf{3}$ & $10.0 \%$ & 14.62 & 19.13 & 11.53 & 13.25 & 12.41 \\
$\mathbf{4}$ & $12.5 \%$ & 12.33 & 15.63 & 9.96 & 11.27 & 15.32 & 10.26 \\
$\mathbf{5}$ & $15.0 \%$ & 11.05 & 13.95 & 9.03 & 10.15 & 9.03 & 12.63 \\
\hline
\end{tabular}

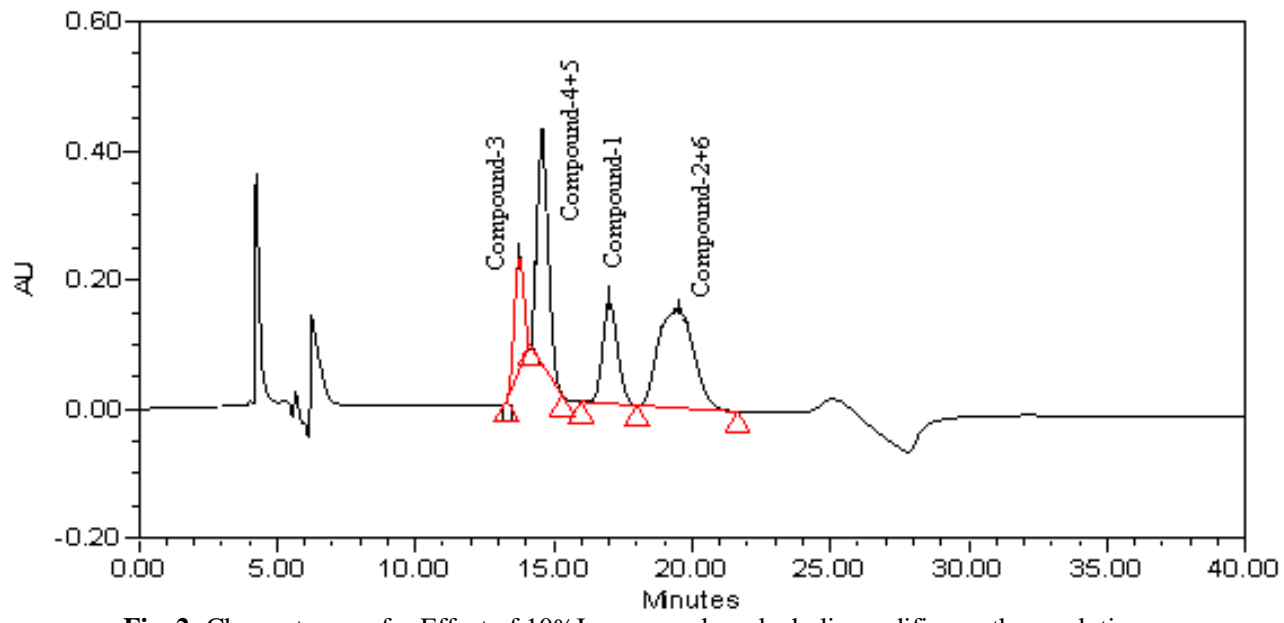

Fig. 2: Chromatogram for Effect of 10\%Isopropanol as alcoholic modifier on the resolutions.

\section{Effect of conc. of Ethanol with $0.1 \%$ each of TEA and TFAA}

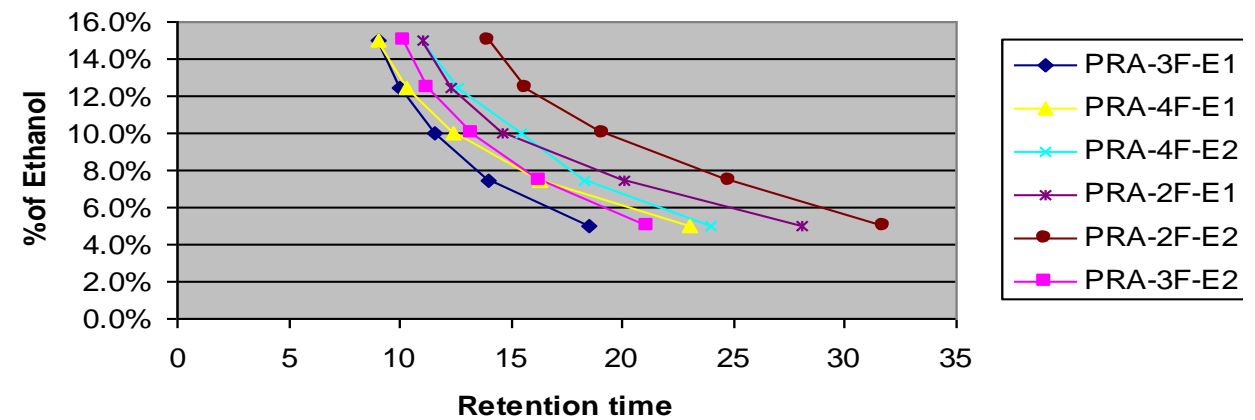

$\mathrm{X}$-axis values: Retention times in minutes, Y-Axis values: \% Ethanol (Polar organic modifier)

Fig. 7: Graphical representation of effect of Ethanol (Polar organic modifier) concentration on retention times.

\section{Effect of basic additive and concentration of organic modifier}

The influence of the basic modifier such as triethyl amine was studied. Addition of $0.1 \%$ triethyl amine (TEA) improved the peak shape by reducing the secondary interactions with the stationary phase but addition of TEA doesn't yielded any significant resolution among the stereo isomers. Effect of ethanol and isopropanol on the resolution and selectivity was also studied. During study, it was observed that the resolutions were dropped and shown broader peaks when isopropanol was used as a polar modifier. At the same time, retentions of the isomer peaks were increased with increase in polar nature of the alcohol, hence ethanol was selected along with both TFA and TEA as additives. Greater than $15 \%$ of ethanol concentration in mobile phase affected the resolution between PRA-4F-E1 (Compound-5) and
PRA-3F-E1(Compound-3) and PRA-E1(Compound-1) and PRA4F-E2(Compound-6). The relationship of enantiomeric resolution (Rs) values with either hydrophobicity or bulkiness of the alcohols, as well as the effect of IPA and ethanol on interaction of the analytes were studied. The best resolution was achieved with the use of $10 \%$ ethanol in the mobile phase. The results are illustrated in Figure-2, Table-3 and figure-7.

\section{CONCLUSIONS}

Baseline separation with resolution greater than 2.0 was achieved between the enantiomers of any regioisomer within 30 min. Refer to Figure-3 and 4, for chromatograms generated with finalized chromatographic conditions. 


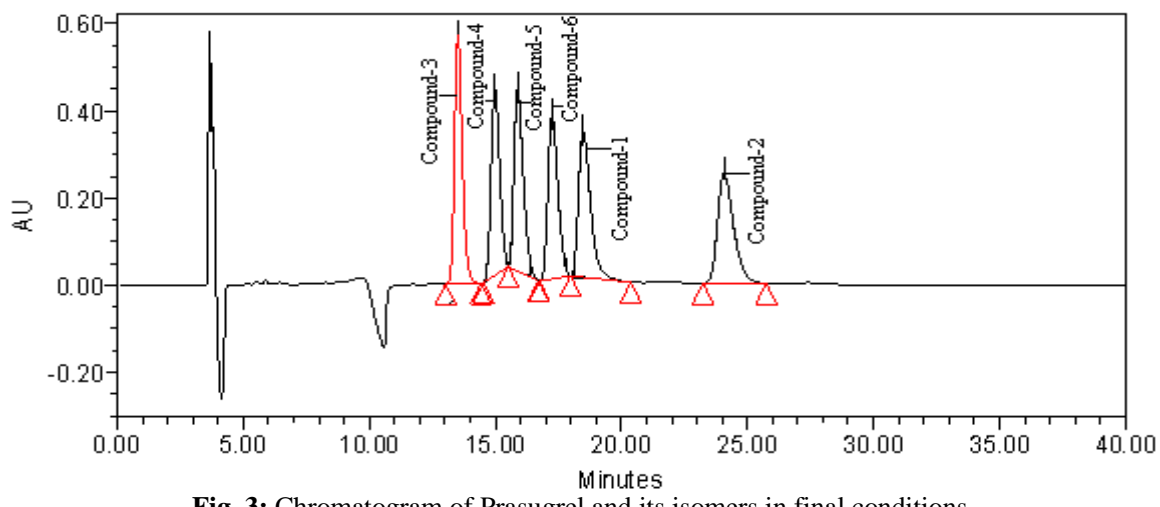

Fig. 3: Chromatogram of Prasugrel and its isomers in final conditions.

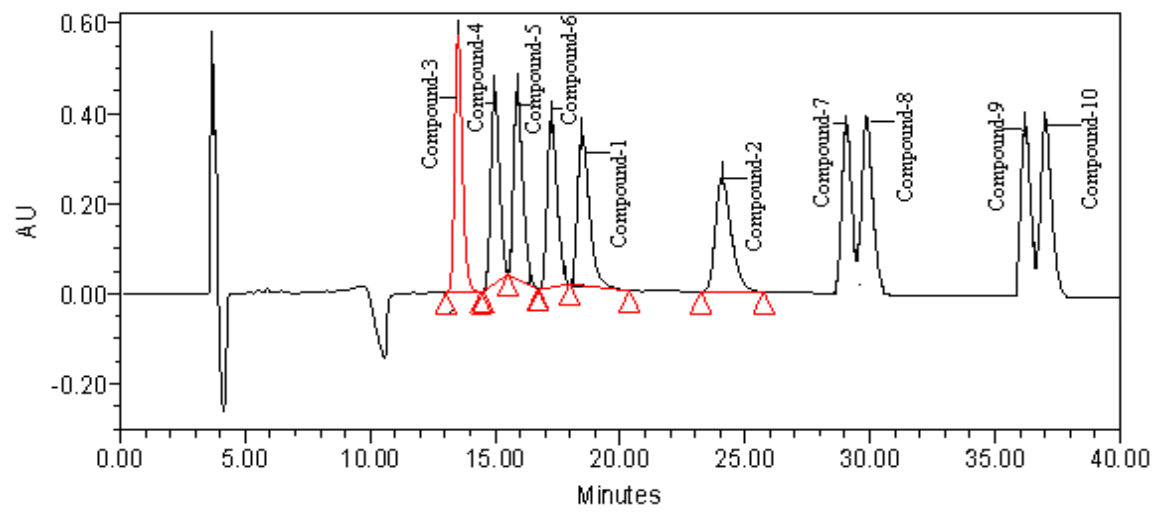

Fig. 4: Chromatogram of Prasugrel, its isomers and degradant (desacetyl impurity) in final conditions.

The effect of organic modifiers and temperature on resolution and retention of enantiomers have been evaluated and the mobile phase composition has been optimized. The results of the study indicates the enantiomeric separation was found to be an enthalpy driven process. Organic modifiers like Isopropanol and polar modifiers like triethylamine and trifluoroacetic acid competes with the solutes for achiral and chiral binding sites. As the steric bulk around the hydroxyl moiety of the modifier plays a role in the mobile phase competition, increased steric bulk resulted in increased k'. However, the results also suggests that the effect of the alcoholic mobile phase modifiers on stereoselectivity may also be due to binding to achiral sites near or at the chiral cavities of the CSP which alters the steric environment of these cavities. This method was designed to determine the relative contribution of each stereoisomer, based on statistical analysis of each stereoisomer's chromatographic peak areas. Although, this work has been done with research purpose and not fully validated, but can be implemented for verification and quality check of chiral impurities of Prasugrel during synthesis, reaction monitoring as well as in finalized Active Pharmaceutical Ingredient.

\section{ACKNOWLEDGEMENTS}

The authors wish to thank the management of Dr. Reddy's Laboratories, IPDO, Hyderabad for supporting this work
(IP Clearance no: IPM - 00291). Also wish to thank Mr.A.Sampath-Process research group for providing the samples for our research and continuous support. The author bears no conflict of interest towards publication of this work.

Conflict of Interests: There are no conflicts of interest.

\section{REFERENCES}

Application notes for Lux amylose-2 columns, http://www.phenomenex.com/lux (accessed 15.09.2016).

Bhatt DL, Topol EJ, Scientific and therapeutic advances in antiplatelet therapy, Nat Rev Drug Discov. 2003; 2(1):15-28.

Enaksha R. Wickremsinhe, Ye Tian, Kenneth JR, Elizabeth MV, Govinda J. Weerakkody, Atsushi K, Nagy A. F, Stereoselective Metabolism of Prasugrel in Humans Using a Novel Chiral Liquid Chromatography-Tandem Mass Spectrometry Method. Drug Metabolism and Disposition. 2007; 35 (6): 917-921. DOI:10.1124/dmd.106.014530.

Gübitz G, Schmid MG, Chiral separation principles in chromatographic and electromigration techniques., Mol Biotechnol. 2006; 32(2):159-80.

Kapendra S, C. Karthikeyan, N. S. Hari NM, Piyush T, Comparative study of forced degradation behaviour of prasugrel by uplc and hplc and the development of validated stability indicating assay method, J. Liq chr reltech, 2011, 34: 1870-1884, DOI: 10.1080/10826076.2011.582209.

Khatuna G, Lali C, Maia T, Tivadar F, Bezhan C, Effect of Basic and Acidic Additives on the Separation of Some Basic Drug Enantiomers on Polysaccharide-Based Chiral Columns With Acetonitrile as Mobile Phase. Chirality, 2015. DOI: 10.1002/chir.22417 
Levin S, Abu-Lafi S, The Role of Enantioselective Liquid Chromatographic Separations Using Chiral Stationary Phases in Pharmaceutical Analysis, Adv Chromatogr 1993., 33, 233-266.

Lien Ai N, Hua H, Chuong Pham-H, Chiral Drugs: An Overview, Int J Biomed Sci. 2006; 2(2): 85-100.

$\mathrm{Lu} \mathrm{H}$, Stereoselectivity in drug metabolism, Expert Opin Drug Metab Toxicol., 2007; 3(2):149-58.

Malati. V, Raghupati R.A , Mukkanti.K, Suryanarayana.M, Identification of prasugrel (an antiplatelet drug) impurities by LC-MS/MS, rapid determination of prasugrel hydrochloride-related substances, and degradation products in active pharmaceutical ingredient and pharmaceutical dosage forms by stability indicating ultra-performance liquid chromatographic method, J. Liq chr reltech, 2012; 36(1), 61-79. DOI : 10.1080/10826076.2011.644052

Matthijs. N, Maftouh M, Vander HY, Screening Approach for Chiral Separation of Pharmaceuticals IV. Polar Organic Solvent Chromatography, Feb 17, J Chromatogr A , 2006, 1111 (1), 48-61. Overview of Prasugrel. https://www.drugs.com/cdi/prasugrel.html, 2016 (accessed 15.08.2016).

Ojikumar L, Mukund Z, Chandan KJ, Shivaji Parmar, Keshav S. Tomar, Amit H, Electrospray ionization LC-MS/MS validated method for the determination of the active metabolite (R-138727) of prasugrel in human plasma and its application to a bioequivalence study, Drug testing analysis, 2011, DOI: 10.1002/dta.264.

Perrin C, Vu VA, Matthijs N, Maftouh M, Massart DL, Vander HY, Screening Approach for Chiral Separation of Pharmaceuticals. Part I. Normal-Phase Liquid Chromatography,. J Chromatogr A, 2002, 947 (1), 69-83.

Stevenson D, Wilson I D, 1991, Recent Advances in Chiral Separations, Switzerland : Chromatographic Society Symposium Series, Springer International Publishing AG.

Singh DK, Sahu A, Handa T, Narayanam M, Singh S, Study of the forced degradation behavior of prasugrel hydrochloride by liquid chromatography with mass spectrometry and liquid chromatography with NMR detection and prediction of the toxicity of the characterized degradation products., J Sep Sci., 2015; 38(17): 2995-3005. doi: $10.1002 /$ jssc. 201500442 .
Ulrich EM, Morrison CN, Goldsmith MR, Foreman WT, Chiral pesticides identification, description, and environmental implications, Rev Environ Contam Toxicol, 2012 ; 217:1-74. doi: 10.1007/978-1-46142329-4_1.

United States Pharmacopeia, 39-NF-34 ed., Rockville, MD: The United States Pharmacopeial Convention.

Waldeck B, Biological significance of the enantiomeric purity of drugs, Chirality. 1993;5(5):350-5.

Wiviott SD, Braunwald E, McCabe $\mathrm{CH}$, Montalescot G, Ruzyllo W, Gottlieb S, Neumann FJ, Ardissino D, De Servi S, Murphy SA, Riesmeyer J, Weerakkody G, Gibson CM, Antman EM; Prasugrel versus Clopidogrel in Patients with Acute Coronary Syndromes, N Engl J Med, 357:2001-2015. Nov 15, 2007, DOI:10.1056/NEJMoa0706482.

Ye Y.K, R.W. Stringham, The effect of mobile phase composition in the enantioseparation of pharmaceutically relevant compounds with polysaccharide-based stationary phases, J. Chromatogr. A 927, 2001.

Z.-M. Wang, J. Zhao and G. Xu, Prasugrel, a new medicine for preventing blockages in the arteries, Acta Cryst. 2010., E66, o1354, DOI :10.1107/S1600536810017095.

\section{How to cite this article:}

Nagireddy V, Vamsikrishna G, Malati V, Ramadevi B, Ravindrakumar Y. Chiral recognition of polysaccharide based CSP for separation of enantiomers and regio isomers of Prasugrel and its related impurities. J App Pharm Sci, 2017; 7 (07): 218-224. 